\title{
Tram-Track Suture Technique for Pupillary Capture of a Scleral Fixated Intraocular Lens
}

\author{
Sung In Kim Kiseok Kim \\ Department of Ophthalmology, Saevit Eye Hospital, Goyang, Korea, Republic of
}

\section{Keywords}

Tram-track suture $\cdot$ Intraocular lens $\cdot$ Pupillary capture

\begin{abstract}
Purpose: To report a new technique using tram-track suture for pupillary capture of a scleral fixated posterior chamber intraocular lens (PC-IOL) to reposition the tilted IOL. Methods: In this prospective interventional case series, we describe a tram-track suture for pupillary capture of a scleral fixated PC-IOL. A long straight needle with double-armed 10-0 polypropylene is passed behind the iris and just above the optic portion (tilted forward) of the IOL. The other straight needle with double-armed 10-0 polypropylene is passed just below the optic portion (tilted backward) of the IOL. After the IOL is repositioned properly, the polypropylene sutures are gently pulled and tied. Results: Four eyes of 4 patients underwent tram-track suture for pupillary capture of a scleral fixated PC-IOL. No intra- or postoperative complications were noted, and no pupillary captures were detected during the follow-up period. Conclusions: The tram-track suture technique provides good centration and stability of a PC-IOL. This technique is an easy and effective way to reposition pupillary capture of an IOL. Further, it is also minimally invasive as it maintains a closed system.




\section{Case Reports in Ophthalmology}

\section{Introduction}

Implantation of a transscleral sutured posterior chamber intraocular lens (PC-IOL) is used in the surgical management of aphakia and inadequate capsular support [1]. In this respect, transscleral sutured PC-IOLs have been shown to be safe and effective. Although the reported incidence of potential complications is low, early postoperative complications such as knot erosion, broken fixation sutures, vitreous hemorrhage, retinal detachment, endophthalmitis, and angle closure glaucoma can occur [2-4]. Also, long-term follow-up is associated with a high rate of postoperative complications requiring further operation.

Tilting of a PC-IOL can occur due to loosening of the polypropylene suture or aqueous flow, and tilting of an IOL can induce pupillary capture of the PC-IOL. Pupillary capture can occur when any portion of a PC-IOL is anterior to the iris [5]. It can reduce vision due to lens tilt, chronic uveitis, deposits on the IOL, and cystoid macular edema [6]. Early management of pupillary capture may prevent these complications and restore a freely mobile pupil [7].

Pupillary capture of PC-IOLs can be managed by exchanging or repositioning the IOL by one-haptic scleral fixation. Exchanging the IOL is more traumatic, requiring large incision and showing an increased risk of vitreous loss, iris trauma, corneal endothelial damage, and postoperative astigmatism than repositioning [8]. Repositioning the IOL by one-haptic scleral fixation is technically demanding and also bears potential risks of iris trauma, endothelial damage, vitreous loss, and retinal detachment.

We introduce a technique using tram-track suture for pupillary capture of a scleral fixated PC-IOL to reposition the tilted IOL.

\section{Surgical Technique}

A long straight needle with double-armed 10-0 polypropylene is introduced into the eye through the sclera $3.0 \mathrm{~mm}$ posterior to the limbus at 8 o'clock.

The needle is advanced behind the iris until it is seen behind the pupil and passed just above the optic portion (tilted forward) of the IOL (fig. 1b).

A 26-gauge needle is passed from the opposite site $(2.0-3.0 \mathrm{~mm}$ posterior to the limbus at 4 o'clock).

After the straight needle is docked into the lumen of a 26 -gauge needle, the 2 needles are removed together from the eye (fig. 1c).

The other straight needle with double-armed 10-0 polypropylene is introduced into the eye through the sclera $3.0 \mathrm{~mm}$ posterior to the limbus at 10 o'clock. Then, the needle is advanced behind the iris until it is seen behind the pupil and passed just below the optic portion (tilted backward) of the IOL.

A 26-gauge needle is passed from the opposite side $(3.0 \mathrm{~mm}$ posterior to the limbus at 2 o'clock). After the straight needle is docked into the lumen of a 26-gauge needle, the 2 needles are removed together from the eye (fig. 1d).

After the IOL is repositioned properly, the polypropylene sutures are gently pulled and tied. The suture knot is left long to prevent knot exposure (fig. 1e). 


\section{Case Reports in Ophthalmology}

\section{Representative Case}

This technique was used in a 47-year-old male patient (case 1) who was seen at our clinic for severe ocular pain and blurred vision in the left eye. He had undergone transscleral suture fixation of a PC-IOL 1 year ago. His vision in the left eye was 20/30 uncorrected, with no improvement on manifest refraction. Intraocular pressure was $18 \mathrm{~mm} \mathrm{Hg}$ in the left eye. Slit-lamp examination of the left eye showed inferior pupil capture of the IOL (fig. 2). Dilated fundus examination showed normal retina and disc in the left eye. Pupillary capture of the IOL was repositioned using tram-track suture.

At the 2-week follow-up visit, the patient was doing well. The vision in his left eye was 20/20 corrected, and the IOL was well centered (fig. 3). At the 1-year follow-up, his left eye vision was 20/20 corrected, and the endothelial cell count remained stable. In addition, there was no suture exposure or IOL tilting. Four eyes with pupillary capture have been operated on (table 1).

\section{Discussion}

Transscleral fixation of a PC-IOL can be used in the surgical management of aphakia and inadequate capsular support [1]. However, tilting of a PC-IOL can occur due to loosening of the polypropylene suture or aqueous flow.

Pupillary capture can occur when any portion of a PC-IOL is anterior to the iris [5]. Tilting of a PC-IOL induces pupillary capture after scleral fixation of the IOL. There is no method to finely control tilted PC-IOL. Current methods for pupillary capture of a scleral fixated IOL include exchanging or repositioning the IOL by one-haptic scleral fixation.

Exchanging or repositioning the IOL induces postoperative astigmatism, transient hypotony, and vitreous loss, bears a risk of dropping the IOL, has a long operation time, and needs longer visual rehabilitation.

The tram-track suture technique prevents pupillary capture from inhibiting an anterior IOL tilt by 2 strings of barrier suture. Our technique does not require corneal incision or extraocular exposure of the IOL. Also, it provides fine tuning of the IOL position and early visual rehabilitation.

Yoo et al. [9] reported that pupillary capture of an IOL was effectively prevented using the H-technique (safety barricade suture) similar to our technique in 40 eyes. They underwent barricade suture and IOL scleral fixation simultaneously. However, we did not remove the IOL, and the IOL tilt was corrected by only suture technique.

Tram-track suture can be used in tilting a PC-IOL without complete breakage of the polypropylene suture after transscleral fixation of thePC-IOL. It can be applied to any form or shapes, such as two strings above the IOL, behind the IOL, as well as above and below the IOL according to the rotation tendency of the IOL. When a problem is found in the orientation and position of the IOL during or after surgery, the tram-track suture can be easily removed and resutured.

Possible complications of our technique include peripheral anterior synechiae and corneal endothelial damage. These can occur when the IOL is pushed too far forward by the strings. The main limitation of the technique is that the long-term stability of the IOL is unknown. 
We think that the tram-track suture technique is minimally invasive as it maintains a closed system. It also provides good centration and stability of the PC-IOL and is an easy and effective way to reposition papillary capture of an IOL.

\section{Statement of Ethics}

The authors have no ethical conflicts to disclose.

\section{Disclosure Statement}

The authors indicate no financial support or any conflicts of interest.

\section{References}

1 Vote BJ, Tranos P, Bunce C, Charteris DG, Da Cruz L: Long-term outcome of combined pars plana vitrectomy and scleral fixated sutured posterior chamber intraocular lens implantation. Am J Ophthalmol 2006;141:308-312.

-2 Wagoner MD, Cox TA, Ariyasu RG, Jacobs DS, Karp CL; American Academy of Ophthalmology: Intraocular lens implantation in the absence of capsular support: a report by the American Academy of Ophthalmology. Ophthalmology 2003;110:840-859.

-3 Johnston RL, Charteris DG, Horgan SE, Cooling RJ: Combined pars plana vitrectomy and sutured posterior chamber implant. Arch Ophthalmol 2000;118:905-910.

-4 Solomon K, Gussler JR, Gussler C, Van Meter WS: Incidence and management of complications of transsclerally sutured posterior chamber lenses. J Cataract Refract Surg 1993;19:488-493.

5 Galvis V, Tello A, Montezuma S: Delayed pupillary capture and noninvasive repositioning of a posterior chamber intraocular lens after pupil dilation. J Cataract Refract Surg 2002;28:1876-1879.

-6 Lindstrom RL, Herman WK: Pupil capture: prevention and management. J Am Intraocul Implant Soc 1983;9:201-204.

-7 Rahman R, Rosen PH: Pupillary capture after combined management of cataract and vitreoretinal pathology. J Cataract Refract Surg 2002;28:1607-1612.

-8 Chung EJ, Kim CY, Koh HJ: Ab externo direct suture technique for dislocated intraocular lens. J Cataract Refract Surg 2007;33:955-958.

-9 Yoo YJ, Kim ET, Heo JW: Safety barricade suture for preventing pupillary capture of intraocular lens with scleral fixation: H-technique. Retina 2016;36:206-210. 

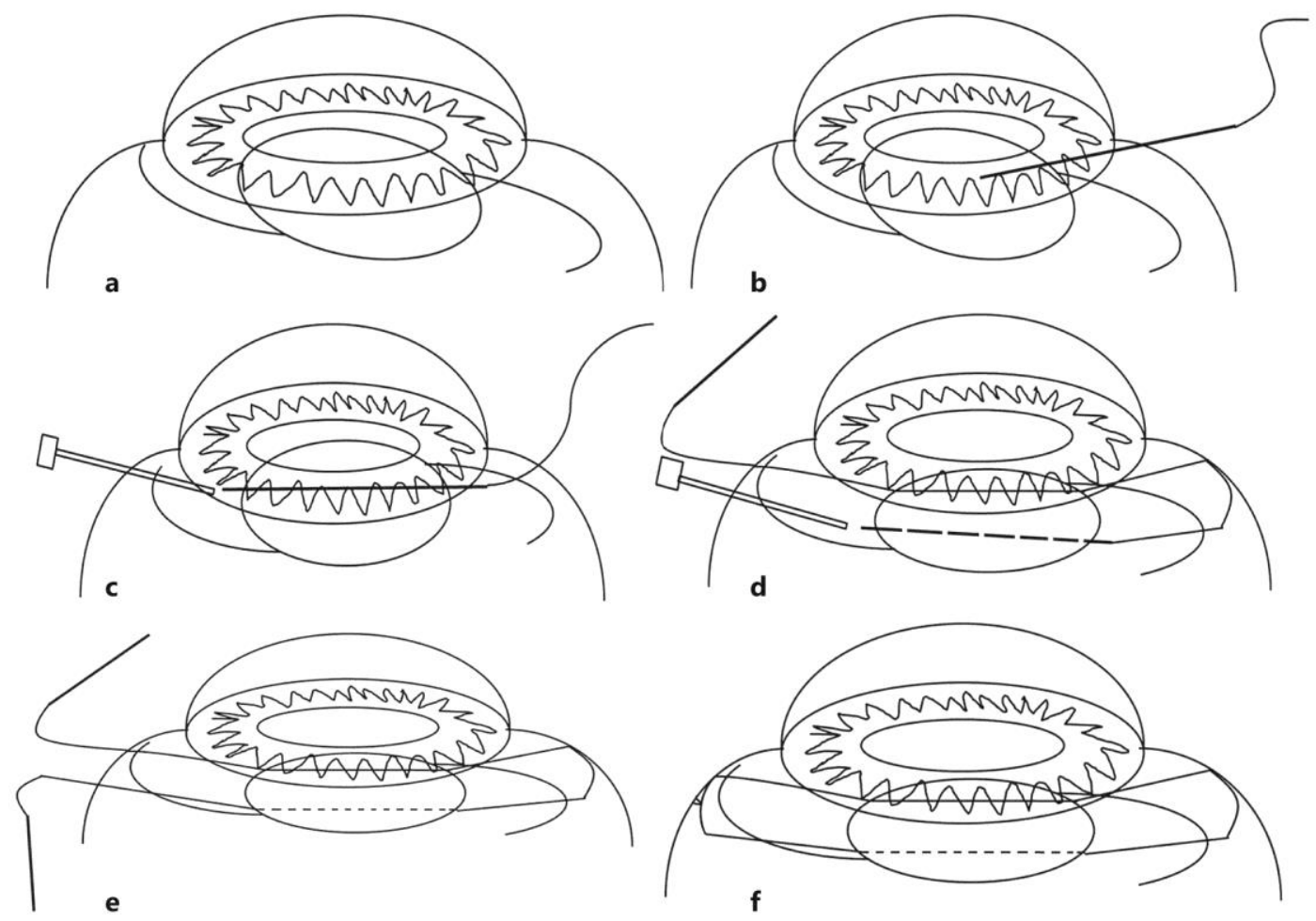

Fig. 1. Tram-track suture technique for pupillary capture of a PC-IOL. a Anterior tilting of the PC-IOL. b A long straight needle with double-armed 10-0 polypropylene is advanced behind the iris and just above the optic portion. $\mathrm{c}$ The needle is docked into the lumen of a 26-gauge needle. $\mathbf{d}$ The other straight needle with double-armed 10-0 polypropylene is passed just below the optic portion of the IOL. e, $f$ After the IOL is repositioned properly, the polypropylene sutures are gently pulled and tied.

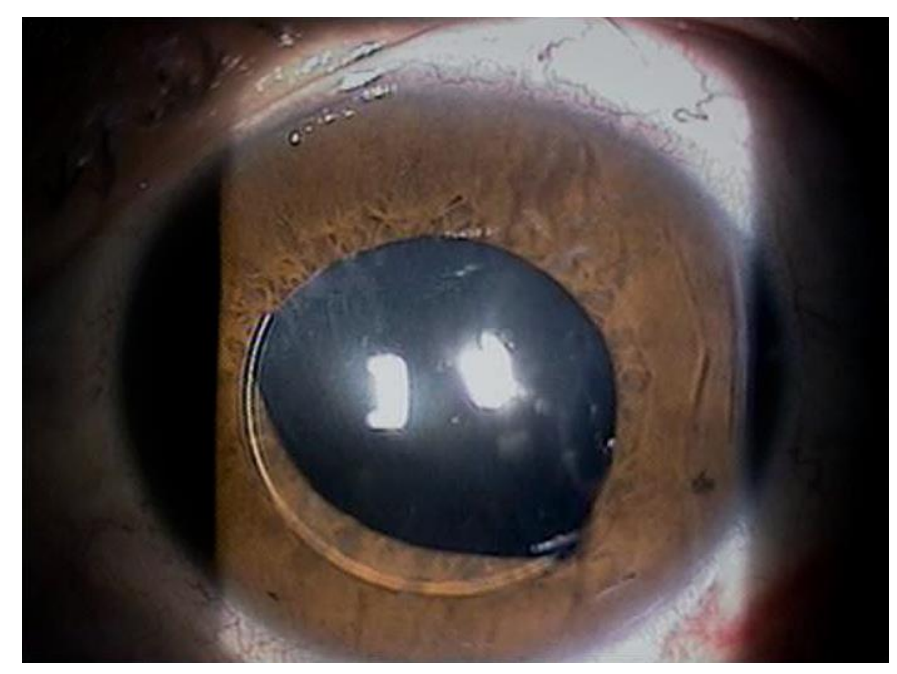

Fig. 2. Pupillary capture of a PC-IOL. 


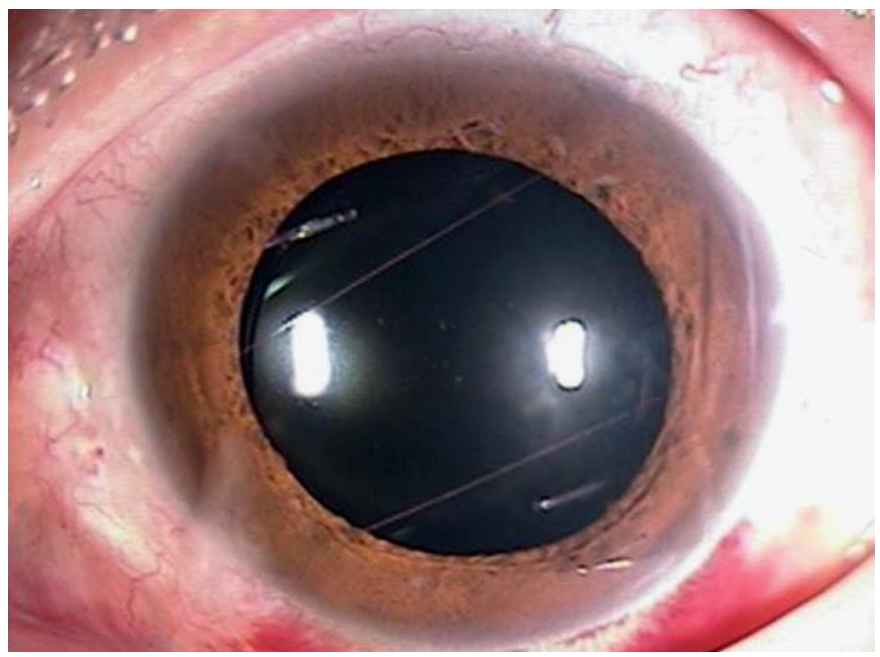

Fig. 3. Slit-lamp photography after the tram-track suture technique.

Table 1. Characteristics of the patients with pupillary capture of a scleral fixated IOL and surgical outcome of tram-track suture

\begin{tabular}{llllllll}
\hline $\begin{array}{l}\text { Case } \\
\text { No. }\end{array}$ & $\begin{array}{l}\text { Sex/ } \\
\text { age, years }\end{array}$ & Eye & $\begin{array}{l}\text { Preop. } \\
\text { BCVA }\end{array}$ & $\begin{array}{l}\text { Postop. } \\
\text { BCVA }\end{array}$ & Preop. refraction & Postop. refraction & $\begin{array}{l}\text { Follow-up, } \\
\text { weeks }\end{array}$ \\
\hline 1 & M/47 & Left & 0.6 & 1.0 & $-0.50:-1.50: 120$ & $-0.75:-0.50: 50$ & 48 \\
2 & M/61 & Left & 0.1 & 0.6 & $-3.00:-2.50: 150$ & $-2.75:-1.25: 100$ & 50 \\
3 & F/49 & Right & 0.4 & 0.8 & $+1.00:-1.50: 140$ & $-1.50:-1.25: 130$ & 47 \\
4 & M/57 & Right & 0.6 & 1.0 & $-0.50:-3.50: 120$ & $-0.50:-2.75: 120$ & 47 \\
\hline
\end{tabular}

Preop. = preoperative; BCVA = best corrected visual acuity; Postop. = postoperative 